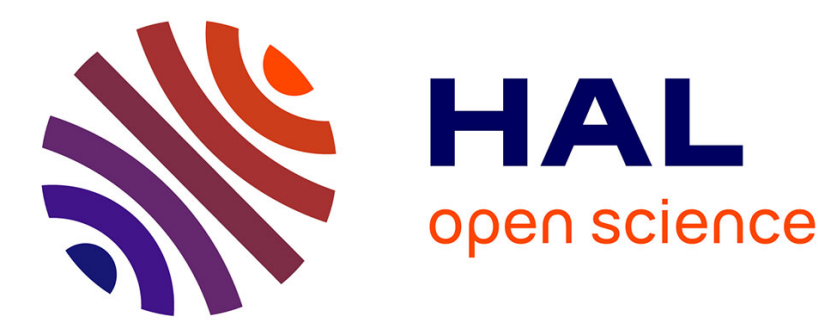

\title{
Optimizing Architectural and Structural Aspects of Buildings towards Higher Energy Efficiency
}

\author{
Álvaro Fialho, Youssef Hamadi, Marc Schoenauer
}

\section{To cite this version:}

Álvaro Fialho, Youssef Hamadi, Marc Schoenauer. Optimizing Architectural and Structural Aspects of Buildings towards Higher Energy Efficiency. GECCO 2011 Workshop on GreenIT Evolutionary Computation, Jul 2011, Dublin, Ireland. inria-00591930

\section{HAL Id: inria-00591930 \\ https://hal.inria.fr/inria-00591930}

Submitted on 10 May 2011

HAL is a multi-disciplinary open access archive for the deposit and dissemination of scientific research documents, whether they are published or not. The documents may come from teaching and research institutions in France or abroad, or from public or private research centers.
L'archive ouverte pluridisciplinaire HAL, est destinée au dépôt et à la diffusion de documents scientifiques de niveau recherche, publiés ou non, émanant des établissements d'enseignement et de recherche français ou étrangers, des laboratoires publics ou privés. 


\section{Optimizing Architectural and Structural Aspects of Buildings towards Higher Energy Efficiency.}

\author{
Álvaro Fialho \\ *LIX, École Polytechnique, \\ Palaiseau, France \\ fialho@lix.polytechnique.fr
}

\author{
Youssef Hamadi \\ *Microsoft Research, \\ Cambridge, United Kingdom \\ *Microsoft Research-INRIA \\ Joint Centre, Orsay, France \\ *LIX, École Polytechnique, \\ Palaiseau, France \\ youssefh@microsoft.com
}

\author{
Marc Schoenauer \\ *Project-Team TAO, INRIA \\ Saclay, Orsay, France \\ *Microsoft Research-INRIA \\ Joint Centre, Orsay, France \\ marc.schoenauer@inria.fr
}

\begin{abstract}
In this on-going work, we aim at contributing to the issue of energy consumption by proposing tools to automatically define some aspects of the architectural and structural design of buildings. Our framework starts with a building design, and automatically optimizes it, providing to the architect many variations that minimize, in different ways, both energy consumption and construction costs. The optimization stage is done by the combination of an energy consumption simulation program, EnergyPlus, with a state-of-the-art multi-objective evolutionary algorithm, Hype. The latter explores the design search space, automatically generating new feasible design solutions, which are then evaluated by the energy simulation software. Preliminary results are presented, in which the proposed framework is used to optimize the orientation angle of a given commercial building and the materials used for the thermal insulation of its walls.
\end{abstract}

\section{Categories and Subject Descriptors}

I.2.8 [Computing Methodologies]: Artificial Intelligence: Problem Solving, Control Methods, and Search

\section{General Terms}

Algorithms

\section{Keywords}

Sustainable development, intelligent building design, energy efficiency, construction costs, multi-objective optimization.

\section{INTRODUCTION}

The continuous rising of energy consumption is a current and global concern. On the one hand, there is the fact that energy is still mainly coming from non-renewable and limited

Permission to make digital or hard copies of all or part of this work for personal or classroom use is granted without fee provided that copies are not made or distributed for profit or commercial advantage and that copies bear this notice and the full citation on the first page. To copy otherwise, to republish, to post on servers or to redistribute to lists, requires prior specific permission and/or a fee.

GECCO'11, July 12-16, 2011, Dublin, Ireland. CACM, 2011. This is the author's version of the work. It is posted here by permission of ACM for your personal use. Not for redistribution. The definitive version will be published in the GECCO'11 Companion proceedings.

Copyright 2011 ACM 978-1-4503-0690-4/11/07 ...\$10.00. sources. On the other hand, the more energy is consumed, the more carbon emissions are released in the atmosphere. According to a recent report from the World Business Council for Sustainable Development 10, the building sector is responsible for the most important energy consumption rate, estimated at around $40 \%$ of the total energy used worldwide. Surprisingly, the resulting carbon emissions are even higher than those of all the transportation sector combined. Reducing energy consumption, while not compromising the rising living standards of the ever-growing population, has become thus a matter of extreme importance towards a global sustainable future. In this work, we aim at contributing to this issue by using evolutionary algorithms to automatically define some aspects of the architectural and structural design of buildings.

The main objective for the optimization of buildings, in terms of sustainable development, is the reduction of energy use (while also possibly generating some energy, e.g., solar or wind energy, but this is out of the scope of this work for the time being). But the more energy-efficient is the building, the more expensive tends to be its construction. One might thus find a compromise between energy efficiency and construction costs. Another well-known trade-off in this context is the exploration of natural daylighting versus thermal insulation. The bigger the windows, the more daylight will possibly come in; but windows are usually much less efficient in terms of thermal insulation than walls. Then, some savings in the use of electricity for lighting might be (probably over) compensated by a higher need of the "Heating, Ventilating and Air-Conditioning" (HVAC) mechanisms, which are usually the most energy-demanding equipments in a building. The non-use of these equipments, or their use at a smaller power rate, might significantly reduce the total energy consumption; but at the same time it will also negatively affect the thermal comfort of the people living/working in the given building.

Based on these examples, it is clear that multiple objectives need to be taken into account in order to do a more realistic optimization of building designs towards sustainability. Evolutionary algorithms are very popular meta-heuristics for multi-objective optimization, mainly due to the fact that they are population-based, i.e., a set of solutions is continuously evolved in parallel. This enables the decision-maker to choose, in the end of the process, between many solutions that are optimal with respect to the considered objectives 
in different ways, not being comparable between each other, the so-called Pareto front.

In this work, the optimization of building designs is done by the combination of an energy consumption simulation program, EnergyPlus 9], with Hype [3, a state-of-the-art multi-objective evolutionary algorithm. The latter explores the design search space, automatically generating new feasible design solutions, which are then evaluated by the energy simulation software. This generate-and-test cycle is repeated until a satisfactory design is found, or another stopping condition is achieved.

This work is on its very early stages. Some preliminary results will be presented here, concerning the definition of the orientation angle of a given commercial building (w.r.t. the real North axis) and the choice of the materials used in two insulation layers of its exterior walls. The objective in this case is to minimize both energy consumption and construction costs. It is important to note that, for the sake of simplicity, we are currently considering only the period during which the building is in "operation". This corresponds to around $80 \%$ of the total energy consumption during the life cycle of a building [10]; the other $20 \%$ are spent on its construction (including the manufacturing of the materials used) and demolition.

This document is structured as follows. Section 2 surveys some related work. In Section 3 the proposed approach is described, including a brief description of both, the EnergyPlus simulation program and the evolutionary algorithm employed. Finally, Section 4 analyzes the results of some preliminary experiments, while Section 5 concludes with some perspectives for further work.

\section{RELATED WORK}

Some other works applying optimization methods to maximize energy efficiency and other sustainability-related objectives can be found in the literature. A brief overview of some of these works will be provided here, focusing on which kind of aspects were optimized, and to which objectives.

In 8, the authors optimize the shape of the building envelope in order to maximize the receiving of solar beams on its façade, which directly affects the energy consumption for artificial lighting and HVAC. Both variables are computed using the EnergyPlus software. As an extra, the authors suggest the use of Computational Fluid Dynamics (CFD) analysis to, for example, analyze the wind flow in the internal space of the building.

In 6], as in the present work, the objective is to minimize costs while maximizing energy efficiency. As choices for the optimization method, there are the type of windows and the type and quantity of the insulation material used in the walls. The thermal transmittance and the conductivity of each material are taken from the ASHRAE database 1], and the prices are artificially simulated according to the performance of the material. Finally, the energy consumption is evaluated using a set of well-defined equations.

In 4, many different applications are considered, with growing levels of complexity, which can be summarized as follows. Starting with box-like offices facing each cardinal direction (squared one-floor buildings), the first objective is to maximize the energy efficiency by finding the best window dimension for each office and orientation. In the second case, the geometry of the building and the space layout are fixed, the objective is to optimize its façade, mainly the size and placement of the windows, aiming at maximizing the energy efficiency. In the following case, the objective is two-fold: minimize the cost of the materials used in the construction of the building, while also maximizing the energy performance. In a second step, not only the cost of the materials is considered, but also the energy saved during the construction phase and the energy spent to manufacture these materials. In the last case, the objective is to automatically generate/evolve complete 3D architectural forms that are energy-efficient, while at the same time being in agreement with the design intentions expressed by the architect, which are represented in terms of a well-defined set of rules.

Besides the building design, another component that significantly affects the energy consumption is the HVAC system. The optimization of the design and of the control parameters of HVAC systems is the focus of the works presented in [1] 2].

\section{OPTIMIZATION FOR SUSTAINABILITY}

In this work, our approach to optimize buildings towards a higher sustainability (here expressed in terms of reduction of energy consumption) can be summarized in this way. A multi-objective evolutionary algorithm explores the design search space, guiding the search towards the Pareto front, according to the required objectives. Two feedbacks are being currently used by the search process: (i) the construction costs of the candidate building design, defined by the price per square meter of the chosen insulation materials, and (ii) its energy efficiency, which is evaluated using a piece of software referred to as EnergyPlus 9]. Both, the EnergyPlus simulation software, and the multi-objective evolutionary algorithm used in this work, will be briefly described in the following.

\subsection{EnergyPlus}

EnergyPlus is a very complete energy analysis and thermal load simulation program, which is availabl】 free of charge for all the main computer platforms. It can be seen as an ameliorated extension of the BLAST and DOE-2 energy simulation programs, that were developed after the 1970s energy crisis. Many enhancements have been continuously aggregated to it since its first release in 2001, mainly supported by the United States Department of Energy.

This software enables the evaluation of the energy consumption behavior of almost any kind of building, as defined by the user via an input file containing the design parameters, and according to the meteorological trends (weather data) of the region where it is planned to be built. It is primarily a simulation engine, with both input and output being made via text files. Some GUIs and special plugins are provided by third-party developers in order to facilitate and extend its use.

It is mainly used by "design engineers" and architects to, for instance, appropriately size the HVAC equipments, develop retrofitting projects, evaluate and/or optimize energy performance, etc. In this work, we use it to evaluate the energy consumption of the design solutions automatically generated by a search/optimization algorithm, which will be described in the following.

$\begin{array}{lc}{ }^{1} \text { EnergyPlus is } & \text { available } \\ \text { http://apps1.eere.energy.gov/buildings/energyplus/ }\end{array}$

at 


\subsection{Multi-Objective Evolutionary Algorithm}

A current and efficient trend to evaluate the quality or fitness of a solution in multi-objective optimization is the use of the hyper-volume measure [12. Briefly, the fitness of a solution is equal to its contribution to the hyper-volume computed between the current Pareto front and a reference point in the search space. In this work, we are using Hype [3], a state-of-the-art hyper-volume-based algorithm.

Besides proposing a new approximate way of calculating the hyper-volume based on sampling, the Hype algorithm 3 differs from the other available variants of hyper-volumebased multi-objective evolutionary algorithms mostly in two aspects: its fitness assignment and its replacement/survival selection mechanism.

Concerning the fitness assignment, as in the other existing algorithms, the fitness assigned to a solution is equal to its contribution to the current hyper-volume. The main difference is that each solution receives partial "credit" for the regions of the hyper-volume that are dominated by more than one solution. For example, in case there are three solutions dominating a given region, the reward given to each of them due to the dominance of this region is divided by three.

Regarding the replacement/survival selection mechanism, Hype proposes a special way of doing it:

1. Starting from the merged parents+offspring population, it firstly divides it into non-dominance partitions, using the non-dominance sorting concept from the NSGAII 5 algorithm. The first partition is the set of nondominated solutions (the Pareto front) of the given population. By removing these solutions from the population, we have a new Pareto front, which is the second partition of non-dominance, and so on. These partitions are then included one by one in the new population, whenever there is space available for the entire partition to be included.

2. At some point, one partition might not entirely fit. It is then considered using the Hype fitness assignment method, as follows. Let $k$ be the number of individuals that need to be removed from this partition so it can be included in the new population. Briefly, at each iteration from 1 to $k$, the fitness (the contribution to the hyper-volume) of each individual of the given subpopulation (the partition) is calculated, and the worst individual is removed.

This process is exemplified in Figure 1 All the solutions are represented by a red cross. The $\times$ marks the first partition of non-dominated solutions, which has 5 individuals, and thus can be entirely included in the new population (of size 8 in this case). The $\times$ marks the second partition (the new Pareto front when removing the first partition). It does not fit entirely in the new population, and only 3 out of 5 individuals are kept, marked with a black square, according to their contribution to the hyper-volume formed by this sub-population.

\section{PRELIMINARY EXPERIMENTS}

This is a currently on-going work, still on its early stages. Some experiments were done in order to validate our experimental framework and to empirically verify the possible

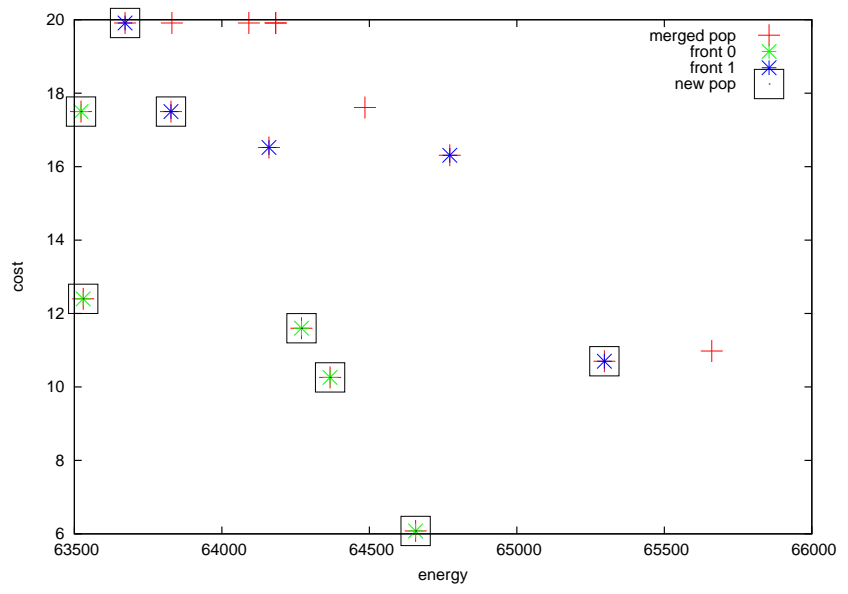

Figure 1: Example of the Hype replacement mechanism in action. The solutions marked with a black square are the ones chosen to remain for the next generation, while the others will be discarded.

gains that might be achieved with it. These experiments will be described in the following.

\subsection{The Building}

The first building design tackled was taken from 7 Exercise $2 \mathrm{C}]$. It is a single-floor commercial building, which is originally described as follows:

Building: Single floor rectangular building 30.5 $m(100 \mathrm{ft}) x 15.2 \mathrm{~m}(50 \mathrm{ft}) .5$ zones -4 exterior, 1 interior, zone height $2.4 \mathrm{~m}(8 \mathrm{ft})$. Exterior zone depth is $3.7 \mathrm{~m}$ (12 ft). There is a 0.6 $m$ (2 ft) high return plenum. The overall building height is $3 \mathrm{~m}$ (10 ft). There are windows on all 4 façades; the south and north façades have glass doors. The south facing glass is shaded by overhangs. The walls are wood-shingle over plywood, R11 insulation, and gypsum board. The roof is a gravel built up roof with mineral board insulation and plywood sheathing. The floor slab is $0.1 \mathrm{~m}$ (4 in) of heavy concrete. The windows and glass doors are double pane Low-e clear glass with argon gap. The window to wall ratio is approximately 0.3. The building is oriented with the long axis running east-west.

Floor Area: 463.6 m2 (5000 ft2)

Internal: Lighting is $16 \mathrm{~W} / \mathrm{m} 2(1.5 \mathrm{~W} / \mathrm{ft2})$, office equipment is $10.8 \mathrm{~W} / \mathrm{m} 2(1.0 \mathrm{~W} / \mathrm{ft} 2) .1 \mathrm{oc}$ cupant per $9.3 \mathrm{~m} 2$ (100 ft2) of floor area. The Infiltration is 0.25 air changes per hour.

HVAC: Single-zone unitary with DX cooling and gas heating serving NORTH PERIMETER zone. $V A V$ with hot water reheat, return plenum, chiller, boiler, and tower serving the other four occupied zones.

Environment: Chicago, IL, USA, TMY2

Different 3D visualizations of this building are shown in Figure 2 


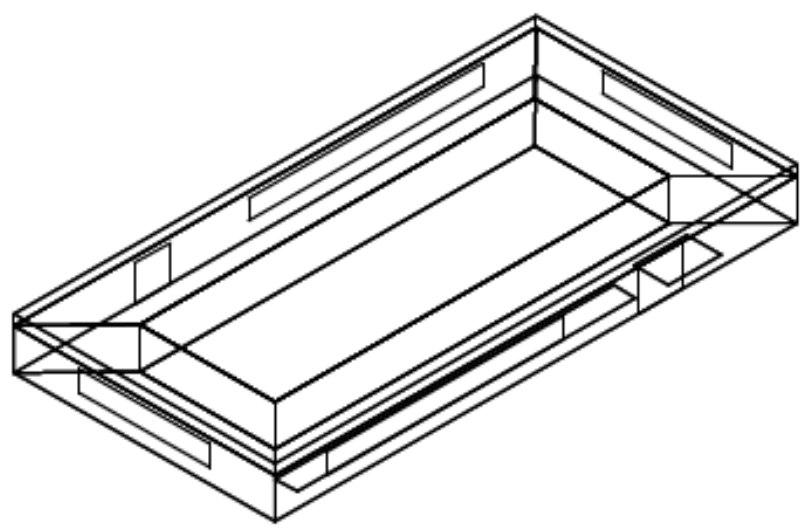

(a) The division of the building in zones, as well as the placement of windows and doors, extracted from [7]

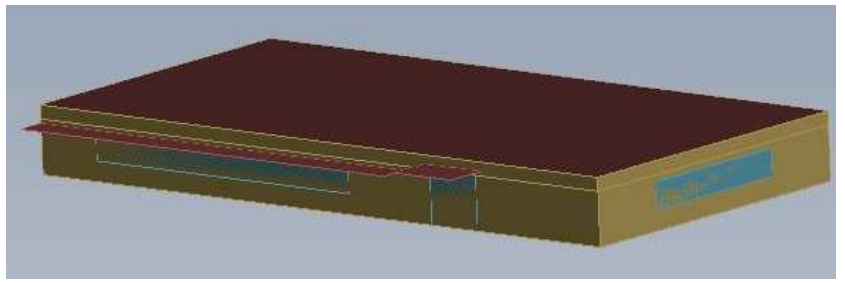

(b) Another view focusing on its external façades

Figure 2: Different views of the building used in the presented experiments.

\subsection{The Problem}

These preliminary experiments are considering two conflicting objectives: the minimization of both the energy consumption and the construction costs of the building. The former is taken from the "Total Site Energy" variable in the "Annual Building Performance Summary" output report provided by EnergyPlus, in kWh. For the latter, we consider the sum of the costs of the insulation materials used, in $€ / m^{2}$. Three problem variables are considered here, as follows.

The first decision variable is the orientation angle of the building, expressed in degrees with respect to the real North axis. It is being currently considered as a real value between 0 and 360 , although in practice some physical constraints might exist.

The other two variables represent the choice of material used for each insulation layer of the external walls. Table 1 depicts our current database of insulation materials, containing 33 different kinds of material with their corresponding thermal resistance (measured in square-meter kelvins per watt, or simply $m^{2} \cdot K / W$ ) and cost per square meter (taken from a french retailer). The names of the materials are omitted here for the sake of brevity. These are thus categorical variables, each one ranging between 1 and 33 (the "index" of each material), with partial ordering: materials \#5 to \#10 might be of the same kind but with different thicknesses (and consequently different thermal insulation performance), while material \#11 might be a totally different one.

As it can be seen from this sample set, the cost is not a function of the thermal resistance (and vice-versa). This is due to the fact that there are other aspects that might make

\begin{tabular}{|c|c|c|}
\hline$\#$ & Cost & ThermalR \\
\hline 1 & 1.67 & 1.10 \\
\hline 2 & 2.30 & 1.50 \\
\hline 3 & 2.42 & 1.53 \\
\hline 4 & 2.48 & 1.50 \\
\hline 5 & 2.62 & 2.00 \\
\hline 6 & 2.62 & 2.00 \\
\hline 7 & 2.63 & 2.00 \\
\hline 8 & 2.65 & 1.26 \\
\hline 9 & 2.67 & 2.50 \\
\hline 10 & 2.72 & 2.04 \\
\hline 11 & 2.73 & 2.50 \\
\hline 12 & 2.77 & 2.55 \\
\hline 13 & 2.97 & 2.50 \\
\hline 14 & 3.12 & 1.20 \\
\hline 15 & 3.35 & 1.20 \\
\hline 16 & 3.87 & 2.10 \\
\hline 17 & 4.08 & 2.00 \\
\hline
\end{tabular}

\begin{tabular}{|c|c|c|}
\hline$\#$ & Cost & ThermalR \\
\hline 18 & 4.58 & 2.00 \\
\hline 19 & 4.71 & 5.10 \\
\hline 20 & 4.74 & 0.55 \\
\hline 21 & 5.35 & 1.10 \\
\hline 22 & 5.82 & 5.00 \\
\hline 23 & 5.99 & 1.15 \\
\hline 24 & 6.24 & 1.20 \\
\hline 25 & 6.34 & 1.60 \\
\hline 26 & 7.64 & 2.15 \\
\hline 27 & 8.55 & 2.65 \\
\hline 28 & 8.92 & 2.35 \\
\hline 29 & 8.95 & 2.65 \\
\hline 30 & 9.73 & 3.15 \\
\hline 31 & 9.97 & 1.75 \\
\hline 32 & 10.18 & 1.80 \\
\hline 33 & 10.18 & 2.40 \\
\hline
\end{tabular}

Table 1: Corresponding cost (in $€ / m^{2}$ ) and thermal resistance $\left(R\right.$ in $\left.m^{2} \cdot K / W\right)$ for each of the insulation materials considered by the optimization process.

a given material more expensive, such as the complexity for its installation, which are not taken into account here. The external walls are finally constituted by the aggregation of these insulation layers with two other layers, the external and the internal ones, which are not modified here in order to avoid possible conflicts with respect to the architect design intentions.

\subsection{Evolutionary Algorithm}

The implemented evolutionary algorithm uses the Hype 3. fitness assignment and replacement mechanisms, briefly described in Section 3.2 The representation used is a mixed one: there are three genes, the first is a real-value representing the orientation angle of the building, and the other two are integer values representing the index of the insulation material used for each layer.

For the mating selection, it uses tournament with $t=$ 2: for the generation of each new solution, two solutions are randomly selected from the main population, and the best between them (in terms of contribution to the hypervolume, as defined by Hype) is selected for the mating. The main population size is fixed to 40 individuals, while 80 new offspring are produced every generation.

Very simple variation operators are being currently employed: a 1-point Crossover, and a Gaussian Mutation with mean zero and standard deviation 1 (multiplied by 10 for the Orientation variable, because it is meaningless to perform very small variations on the building orientation). Crossover is applied at a fixed rate of 0.8 , and Mutation at 0.1 , an identical copy of the solution is created otherwise.

It is important to note that better parameters/operators with respect to the considered search space might exist. But the objective of the presented work is rather to validate the experimental framework and show the potentials of this kind of application. An extensive parameter analysis is left for further work.

The stopping condition is fixed to 6040 evaluations, that is equivalent to 75 generations. A much higher number of gen- 
erations could be considered, but this choice is constrained by the fact that each energy consumption simulation, which is required for each fitness evaluation, takes around 1 minute to be processed 2 .

\subsection{Analysis of Results}

Forty runs were done, considering "cost versus energy consumption". The final population for each of these runs, as well as the non-dominated set extracted from these 40 populations, are presented in Figure 3 The same set of final non-dominated solutions is numerically depicted in Table 2

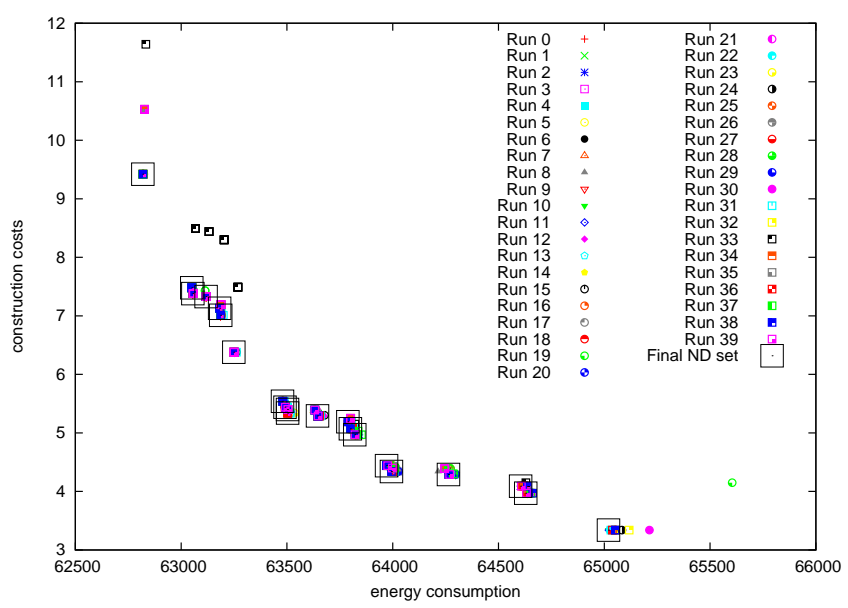

Figure 3: The final population of 40 individuals for each of the 40 runs done. The non-dominated solutions between all these individuals are marked with a black square.

For the sake of comparison, the original design of this building, using Orientation=0 (i.e., aligned with the real North axis) and the materials cited on its description in Section 4.1 achieves a total energy consumption of 65735.2 $\mathrm{kWh}$ per year, considering the meteorological data of the OHare International Airport in Chicago, USA (the same used in these experiments). In case there are no budget constraints, the most energy efficient solution in this case (\#1) will represent a reduction of around 5\% in terms of energy consumption.

These results become more interesting when considering both objectives. As it can be seen, the proposed experimental framework succeeds into finding many non-dominated solutions (21 in this case) that are optimal in different ways with respect to the considered objectives. Then, it is up to the decision maker to choose which solution to employ, according to the strongest requirements and/or constraints he needs to take into account. For instance, if he does not want to spend more than $€ 7$ per square meter for the insulation materials, solution \#7 would be the most energy-efficient choice. On the other way round, if he wants a building that does not spend more than $63500 \mathrm{kWh}$ in average per year, solution \#9 would be the cheapest one.

It is important to note that all these solutions use around the same value for the orientation angle of the building $(\Theta=264.935 \pm 0.177)$. This is intuitive, and now empirically verified: if we use the same combination of materials

${ }^{2}$ In a computer with an Intel Xeon 2,33 GHz CPU and 16 Gb of RAM.

\begin{tabular}{|c|c|c|c|c|c|}
\hline$\#$ & Angle & M1 & M2 & Energy $\Downarrow$ & Cost \\
\hline 1 & 265.11 & 19 & 19 & 62819.3 & 9.42 \\
\hline 2 & 265.141 & 12 & 19 & 63050.3 & 7.48 \\
\hline 3 & 265.153 & 19 & 9 & 63055.4 & 7.38 \\
\hline 4 & 264.925 & 19 & 5 & 63118 & 7.33 \\
\hline 5 & 265.154 & 19 & 3 & 63182 & 7.13 \\
\hline 6 & 265.161 & 19 & 2 & 63186.8 & 7.01 \\
\hline 7 & 265.096 & 19 & 1 & 63248.8 & 6.38 \\
\hline 8 & 265.031 & 12 & 12 & 63478.9 & 5.54 \\
\hline 9 & 265.027 & 12 & 9 & 63490.7 & 5.44 \\
\hline 10 & 264.973 & 11 & 9 & 63502.6 & 5.4 \\
\hline 11 & 264.964 & 9 & 9 & 63503.4 & 5.34 \\
\hline 12 & 264.948 & 6 & 9 & 63644.8 & 5.29 \\
\hline 13 & 264.907 & 12 & 3 & 63788 & 5.19 \\
\hline 14 & 264.904 & 12 & 2 & 63799.9 & 5.07 \\
\hline 15 & 264.87 & 9 & 2 & 63820.9 & 4.97 \\
\hline 16 & 264.839 & 12 & 1 & 63970.7 & 4.44 \\
\hline 17 & 264.839 & 1 & 9 & 63994 & 4.34 \\
\hline 18 & 264.734 & 5 & 1 & 64263.7 & 4.29 \\
\hline 19 & 264.634 & 3 & 1 & 64604.3 & 4.09 \\
\hline 20 & 264.651 & 2 & 1 & 64628.9 & 3.97 \\
\hline 21 & 264.58 & 1 & 1 & 65020 & 3.34 \\
\hline
\end{tabular}

Table 2: The set of non-dominated solutions extracted from the final population of each of the 40 runs done, ordered by the yearly average energy consumption, in $\mathrm{kWh}$, while the cost is shown in $€ / \mathrm{m}^{2}$. M1 and M2 refers to the index of the material respectively used for each of the two insulation layers, following the list in Table 1

for all the external walls of the building, the optimization of the orientation angle becomes an independent problem. In order to further analyze it, additional experiments were performed considering solution \#1 (the most energy-efficient one), varying the angle $\in[0: 5: 360]$. The results are shown in Figure 4 As it can be seen, in this simple study case, the optimum is really around 265 degrees, and up to $700 \mathrm{kWh}$ (around 1\%) of energy consumption can be saved only by optimizing the orientation angle.

Although it is common (and good) practice to compare the obtained results with the ones found by other algorithms, we consider that it is not really necessary to do so for the time being, mainly for three reasons. Firstly, the Hype algorithm has already been empirically shown to be a state-of-the-art approach for multi-objective optimization 3, although in a different application context. Secondly, the experiments presented here are considering a rather simple search space: this is evidenced by the fact that very few points can be seen in Figure 3 which indeed depicts all the 40 final solutions for each of the 40 runs done - this means that most of the runs converged to approximately the same solution set, although using very different random seeds. And thirdly, the main baseline for comparison here is still the human (an architect in this case). Needless to say, besides saving a significant amount of his time, the use of computer-guided optimization enables the exploration of a much higher number of candidate design solutions.

\section{CONCLUSION AND NEXT STEPS}

In this work, we are using an existing multi-objective algorithm, referred to as Hype [3], to optimize some aspects of 


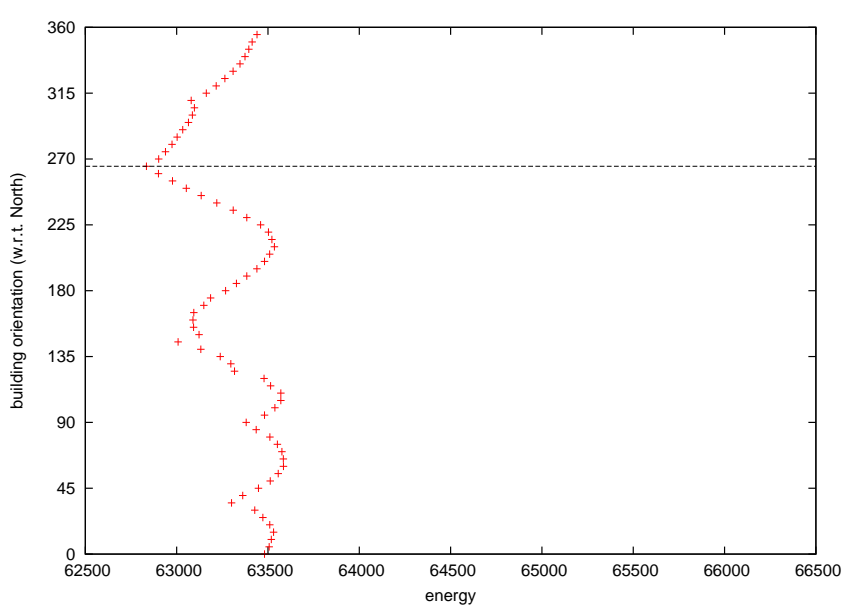

Figure 4: Energy consumption evaluation, in $\mathrm{kW} / \mathrm{h}$ averaged over an year, for different orientation angles of the most energy-efficient building.

the design of a given building. The aim of the optimization process is two-fold: to reduce the energy consumption of the building, given by a simulation engine called EnergyPlus 9], while also accounting for its construction costs.

The experiments presented, although being very preliminary, show to be very useful in order to validate the proposed experimental framework. They also contribute into demonstrating that this project has a great potential in terms of contribution to sustainable development. In addition to the "green" motivation, an architect might greatly benefit from this framework: it will not only save his time, but also provide more energy-efficient solutions that he would probably not be able of exploring.

The complexity of the search space will be significantly increased in the near future, with the consideration of other design aspects, such as the size and placement of the windows. In this case, an additional objective shall be considered, the absorptance of natural daylighting. Additionally, for the time being, we are currently taking into account only objectives related to the reduction of energy consumption in a passive way. Further work might also address the choice of HVAC systems that should be used, as well as their control parameters.

Finally, for the moment we have considered only a single and simple building, while many other buildings should be tackled in order to further validate our approach. The plan is to create a benchmark set containing several building of very different nature, such as office buildings, warehouses, churches, hospitals, schools, with different geometries (e.g., L-shape, U-shape), number of floors, HVAC systems, etc.

\section{Acknowledgements}

We acknowledge here the financial support given by the Microsoft-CNRS Chair on Optimization for Sustainable Development at LIX, École Polytechnique, Palaiseau, France.

\section{REFERENCES}

[1] American Society of Heating, Refrigerating and Air-Conditioning Engineers. ASHRAE Handbook of Fundamentals. ASHRAE, 2009.
[2] P. Angelov, Y. Zhang, J. Wright, V. Hanby, and R. Buswell. Automatic design synthesis and optimization of component-based systems by evolutionary algorithms. In E. Cantú-Paz et al, editor, Proc. GECCO, volume 2724 of $L N C S$, pages 1938-1950. Springer, 2003.

[3] J. Bader and E. Zitzler. Hype: An algorithm for fast hypervolume-based many-objective optimization. Evolutionary Computation, 19(1):45-76, 2011.

[4] L. Caldas. Generation of energy-efficient architecture solutions applying gene_arch: An evolution-based generative design system. Advanced Engineering Informatics, 22(1):59 - 70, 2008.

[5] K. Deb, A. Pratap, S. Agarwal, and T. Meyarivan. A fast and elitist multiobjective genetic algorithm: NSGA-II. IEEE Trans. Evol. Comput., 6:182-197, 2002.

[6] C. Diakaki, E. Grigoroudis, and D. Kolokotsa. Towards a multi-objective optimization approach for improving energy efficiency in buildings. Energy and Buildings, 40(9):1747 - 1754, 2008.

[7] EnergyPlus. Getting started with EnergyPlus. Technical report, Lawrence Berkeley National Laboratory, 2010.

[8] M. Pitman and A. King. Engineering solutions to optimise the design of carbon-neutral tall office buildings. In Proc. International Conference on Solutions for a Sustainable Planet, 2009.

[9] R. Strand, D. Crawley, C. Pedersen, R. Liesen, L. Lawrie, F. Winkelmann, W. Buhl, Y. Huang, and D. Fisher. EnergyPlus: A new-generation energy analysis and load calculation engine for building design. In Proc. Association of Collegiate Schools of Architecture Technology Conference, 2000.

[10] World Business Council for Sustainable Development. Transforming the market: energy efficiency in buildings. Technical report, WBCSD, 2009.

[11] J. Wright, H. Loosemore, and R. Farmani. Optimization of building thermal design and control by multi-criterion genetic algorithm. Energy and Buildings, 34(9):959 - 972, 2002.

[12] E. Zitzler and L. Thiele. Multiobjective optimization using evolutionary algorithms - a comparative case study. In Proc. PPSN V, pages 292-304. Springer, 1998. 